\title{
NUMERICAL INVESTIGATION OF ION TRANSPORT IN UNDER-EXPANDED JET FLOWS
}

\author{
M. Jugroot* and C. P. T. Groth ${ }^{\dagger}$ \\ University of Toronto Institute for Aerospace Studies \\ Toronto, Ontario, M3H 5T6, Canada \\ B. A. Thomson, V. Baranov and B. A. Collings \\ $M D S$ SCIEX \\ Concord, Ontario, L4K \&V8, Canada
}

\begin{abstract}
The transport of free ions through highly under-expanded jet flows of neutral gases and in the presence of applied electric fields is investigated by continuum-based numerical simulations. A five-moment mathematical model and parallel multi-block numerical solution procedure is developed for predicting the ion transport. The model incorporates the effects of ion-neutral collision processes and is used in conjunction with a NavierStokes model and flow solver for the neutral gas to examine the key features of the ion motion. The influences of the neutral gas flow, electric field, and flow field geometry on ion mobility are all carefully assessed. Numerical results are given which are relevant to the ion flows occurring in the interface regions of mass spectrometer systems.
\end{abstract}

\section{Introduction}

$\mathbf{T}$ HE ability to accurately predict and thereby understand the often complex transport of a charged gas through a background neutral gas subject to electro-magnetic forces is very important to furthering the understanding of many advanced technological and industrial plasma processes including those associated with vapour deposition in material and semiconductor processing, ${ }^{1,2}$ microsystems,${ }^{3}$ electric space propulsion devices, ${ }^{4-7}$ and ionospheric and space plasmas processes. ${ }^{8,9}$ The transport of ions through rapidly expanding and/or jet flows are also important to the operation of mass spectrometers, such as liquid chromatography (LC) / mass spectrometry (MS) systems used extensively in the trace analysis of biological fluids for drugs, metabolites, and natural biopolymers. ${ }^{10}$ The latter is the particular application of interest here. The LC/MS instruments make use of an atmospheric pressure ionization technique whereby sample ions are generated at atmospheric pressure from molecules which are contained in micro-droplets and the resulting ions are then transported from the atmospheric pressure conditions into a high vacuum system for spectroscopic analysis. As might be expected, the performance of the mass spectrometers is highly dependent on the ion gas transport from the ion source

\footnotetext{
*Postdoctoral Fellow, jugroot@utias.utoronto.ca

${ }^{\dagger}$ Assistant Professor, Senior Member AIAA, groth@utias. utoronto.ca
}

region to the mass detectors and gaining an improved understanding of ion-source jet flows and related transport phenomena is an active area of research.

This study is concerned with the application of modern numerical methods to the modelling of the transport of free ions through highly under-expanded jet flows of neutral gases in the presence of applied electric fields. A five-moment continuum-based model and parallel multi-block numerical solution procedure is developed and described for predicting the ion transport. The five-moment model incorporates the effects of ion-neutral collision processes and is used in conjunction with a Navier-Stokes model and flow solver for the neutral gas to examine the key features of the ion motion. The influences of the neutral gas flow, electric field, and flow field geometry on ion mobility are assessed and numerical results are given relevant to ion flows occurring in the interface regions of mass spectrometer systems. The capability of controlling the charged particle motions through a combination of directed neutral flow and applied electric field is demonstrated for high-speed, hypersonic, jet flows.

\section{Governing Equations \\ Five-Moment Ion Transport Model}

The transport of a mixture of a neutral gas and free ions is considered here. This mixture is not formally treated as plasma, but rather as a source of positively charged ions, for it is assumed that there are no free electrons (plasmas are comprised of neutral particles, ions, and electrons and the charged particles transport is generally tightly coupled by electric/magnetic ap-

1 
plied/induced fields). For the purposes of this study, it is also estimated and assumed that the ion number density is very low compared with that of the neutral gas such that the ions have a negligible effect on the neutral particles. Hence, the solution of the ion and neutral gas flows can be decoupled. The neutral gas flow field can be predetermined and then the prediction of the ion motion can be carried out using this calculated neutral gas solution. A five-moment closure continuum approximation is used herein to model the motion of the ions through the more dense neutral gas. ${ }^{11-14}$ This mathematical description is a single-temperature, near-equilibrium model that can take into account the effects of ion-neutral collision processes and applied external electric and magnetic forces. Ion self collisions (ion-ion collision processes) are neglected in this approximation and hence the ion fluid stresses and heat flux are assumed to be unimportant.

The governing transport equations of the fivemoment model reflect the conservation of mass, momentum, and energy and can be written as in nonconservative coordinate-free form as

$$
\begin{gathered}
\frac{\partial \rho_{i}}{\partial t}+\nabla \cdot\left(\rho_{i} \mathbf{v}_{i}\right)=0 \\
\frac{\partial \mathbf{v}_{i}}{\partial t}+\left(\mathbf{v}_{i} \cdot \nabla\right) \mathbf{v}_{i}+\frac{1}{\rho_{i}} \nabla p_{i}=\frac{Q_{i}}{m_{i}} \mathbf{E} \\
+\frac{Q_{i}}{m_{i}}\left(\mathbf{v}_{i} \times \mathbf{B}\right)+\sum_{s} \nu_{i s}\left(\mathbf{v}_{s}-\mathbf{v}_{i}\right), \\
\frac{\partial p_{i}}{\partial t}+\left(\mathbf{v}_{i} \cdot \nabla\right) p_{i}+\gamma_{i} p_{i}\left(\boldsymbol{\nabla} \cdot \mathbf{v}_{i}\right)=\frac{\delta p_{i}}{\delta t}
\end{gathered}
$$

where $\rho_{i}=m_{i} n_{i}$ is the ion mass density, $n_{i}$ is the ion number density, $m_{i}$ is the mass of the ion molecules, $\mathbf{v}_{i}$ is the ion velocity, $p_{i}=\rho_{i} R_{i} T_{i}=n_{i} k T_{i}$ is the ion pressure, $T_{i}$ is the ion temperature, $R_{i}$ is the ion ideal gas constant, $k$ is the Boltzmann constant, $\gamma_{i}$ is the ion specific heat ratio, $Q_{i}$ is the ion particle charge, and $\mathbf{E}$ and $\mathbf{B}$ are the external electric and magnetic fields. Ion-neutral collision processes are modelled here using the so-called relaxation-time or BGK (Bhatnagar, Gross, and Krook) approximation for the Boltzmann collision integral. ${ }^{11,13-15}$ The influences of the ion-neutral collisional processes manifest themselves as source terms in the ion momentum and energy equations, Eqs. (2) and (3), respectively. These terms involve a sum over all neutral gas species, $s$, and depend on the the momentum exchange collision frequency, $\nu_{i s}$. The energy or pressure source term, $\delta p_{i} / \delta t$, is given by

$$
\begin{aligned}
\frac{\delta p_{i}}{\delta t}=\sum_{s} \frac{\rho_{i} \nu_{i s}}{m_{i}+m_{s}} & {\left[2 k\left(T_{s}-T_{i}\right)\right.} \\
+ & \left.\frac{2}{3} m_{s}\left|\mathbf{v}_{s}-\mathbf{v}_{i}\right|^{2}\right],
\end{aligned}
$$

where $T_{s}$ and $\mathbf{v}_{s}$ are the temperature and velocity of neutral species $s$, and $m_{s}$ is the mass of the neutral species molecules. The collision frequency, $\nu_{i s}$, can be related to the product of the collision cross section $\sigma_{i s}$, neutral species number density, $n_{s}$, and relative speed of the colliding particles $g_{i s}$. By utilizing the approximation that $g_{i s}=\sqrt{\bar{v}_{i}^{2}+\bar{v}_{s}^{2}}=$ $\sqrt{8 k T_{i} / \pi m_{i}+8 k T_{s} / \pi m_{s}}$, the following expression can be obtained and used to prescribe the ion-neutral collision frequency: ${ }^{14}$

$$
\begin{aligned}
\nu_{i s} & =\sigma_{i s} n_{s} g_{i s} \\
& =\sigma_{i s} n_{s} \sqrt{1+\frac{m_{s} T_{i}}{m_{i} T_{s}}} \sqrt{\frac{8 k T_{s}}{\pi m_{s}}} .
\end{aligned}
$$

The relaxation time model is only an approximation to the Boltzmann collision integral and ignores the detailed nature of inter-particle interactions. Nevertheless, it is thought to be sufficient for the present study of ion transport phenomena.

For a two-dimensional axisymmetric coordinate system, a neutral gases consisting of a single neutral species $s=n$, and negligible magnetic fields $(\mathbf{B}=0)$, the conservative form of the five-moment equations governing ion transport can be summarized as follows:

$$
\frac{\partial \mathbf{U}_{i}}{\partial t}+\frac{\partial \mathbf{F}_{i}}{\partial z}+\frac{\partial \mathbf{G}_{i}}{\partial r}=\mathbf{S}_{\mathbf{a} i}+\mathbf{S}_{\mathbf{e} i}+\mathbf{S}_{\mathbf{c} i},
$$

where $\mathbf{U}_{i}$ is the conserved variable solution vector given by

$$
\mathbf{U}_{i}=\left[\begin{array}{llll}
\rho_{i}, & \rho_{i} u_{i}, & \rho_{i} v_{i}, & \rho \epsilon_{i}
\end{array}\right]^{T},
$$

$z$ and $r$ are the axial and radial spatial coordinates of the axisymmetric frame, $u_{i}$ and $v_{i}$ are the ion axial and radial velocity components, $\epsilon_{i}=p_{i} /\left(\rho_{i}\left(\gamma_{i}-1\right)\right)+$ $\left|\mathbf{v}_{i}\right|^{2} / 2$ is the specific total energy, $\mathbf{F}_{i}$ and $\mathbf{G}_{i}$ are the axial- and radial-direction solution flux vectors given by

$$
\mathbf{F}_{i}=\left[\begin{array}{c}
\rho_{i} u_{i} \\
\rho_{i} u_{i}^{2}+p_{i} \\
\rho_{i} u_{i} v_{i} \\
u_{i}\left(\rho_{i} \epsilon_{i}+p_{i}\right)
\end{array}\right], \mathbf{G}_{i}=\left[\begin{array}{c}
\rho_{i} v_{i} \\
\rho_{i} u_{i} v_{i} \\
\rho_{i} v_{i}^{2}+p_{i} \\
v_{i}\left(\rho_{i} \epsilon_{i}+p_{i}\right)
\end{array}\right],
$$

and $\mathbf{S}_{\mathbf{a} i}, \mathbf{S}_{\mathbf{e} i}$, and $\mathbf{S}_{\mathbf{c} i}$ are source vectors associated with the axisymmetric coordinate frame, electric fields, and ion-neutral collision processes, respectively. The latter are given by

$$
\mathbf{S}_{\mathbf{a} i}=-\frac{1}{r}\left[\begin{array}{c}
\rho_{i} v_{i} \\
\rho_{i} u_{i} v_{i} \\
\rho_{i} v_{i}^{2} \\
v_{i}\left(\rho_{i} \epsilon_{i}+p_{i}\right)
\end{array}\right],
$$




$$
\begin{gathered}
\mathbf{S}_{\mathbf{e} i}=\left[\begin{array}{c}
0 \\
\rho_{i} \frac{Q_{i}}{m_{i}} E_{z} \\
\rho_{i} \frac{Q_{i}}{m_{i}} E_{r} \\
Q_{i} \\
\rho_{i} \frac{u_{i}}{m_{i}}\left(u_{i} E_{z}+v_{i} E_{r}\right)
\end{array}\right] \\
\mathbf{S}_{\mathbf{c} i}=\left[\begin{array}{c}
0 \\
\frac{\rho_{i} m_{n} \nu_{i n}}{m_{i}+m_{n}}\left(u_{n}-u_{i}\right) \\
\frac{\rho_{i} m_{n} \nu_{i n}}{m_{i}+m_{n}}\left(v_{n}-v_{i}\right) \\
\frac{\delta p_{i}}{\delta t}+\frac{\rho_{i} m_{n} \nu_{i n}}{m_{i}+m_{n}}\left[u_{i}\left(u_{n}-u_{i}\right)\right. \\
\left.+v_{i}\left(v_{n}-v_{i}\right)\right]
\end{array}\right],
\end{gathered}
$$

where $E_{z}$ and $E_{r}$ are the axial and radial components of the electric field and $u_{n}$ and $v_{n}$ are the axial and radial components of the neutral gas velocity. Note the presence of Joule heating terms associated with the applied electric field in the source terms of the energy equation. The equation set of (6) is used here to predict ion particle motion through a high-speed neutral gas.

For low-speed ion flows through a single-species, stationary, neutral gas (neglecting inertial effects), the ion drift velocity can be directly related to the applied electric field. It follows from the ion momentum equation that

$$
\mathbf{v}_{i}=\frac{Q_{i}}{m_{i} \nu_{i n}} \mathbf{E}=\eta_{i n} \mathbf{E},
$$

where $\eta_{i n}=Q_{i} / m_{i} \nu_{i n}$ is the ion mobility. It is readily apparent from this expression that the BGK collision model leads to the usual linear relationship between the drift velocity and electric field.

\section{Navier-Stokes Equations for Neutral Gas Flow}

A near-thermal-equilibrium continuum model is also used to describe the neutral gas flows considered in this study. The neutral gas flows are assumed to be laminar and neutral gas is taken to be both calorically and thermally perfect and to obey the ideal gas equation of state, $p_{n}=\rho_{n} R_{n} T_{n}$, where $p_{n}$ is the static pressure of the neutral gas, $\rho_{n}=m_{n} n_{n}$ is the neutral gas mass density, $n_{n}$ is the neutral gas number density, $T_{n}$ is the neutral gas temperature, and $R_{n}$ is the gas constant. Due to the low concentration of ions particles relative the concentration of neutral molecules, ion-neutral inter-particle interactions have an insignificant influence on the motion of the neutral gas. It is therefore appropriate to assume that the neutral gas flow is unaffected by the ion particles. Accordingly, the well-known standard Navier-Stokes equations for a compressible fluid govern the transport of the neutral gas. The conservation form of these equations can be expressed as

$$
\begin{gathered}
\frac{\partial \rho_{n}}{\partial t}+\boldsymbol{\nabla} \cdot\left(\rho_{n} \mathbf{v}_{n}\right)=0, \\
\frac{\partial}{\partial t}\left(\rho_{n} \mathbf{v}_{n}\right)+\boldsymbol{\nabla} \cdot\left(\rho_{n} \mathbf{v}_{n} \mathbf{v}_{n}+p_{n} \mathbf{I}-\boldsymbol{\tau}_{n}\right)=\rho_{n} \mathbf{f}, \\
\frac{\partial}{\partial t}\left(\rho_{n} \epsilon_{n}\right)+\boldsymbol{\nabla} \cdot\left[\mathbf{v}_{n}\left(\rho_{n} \epsilon_{n}+p\right)+\mathbf{q}_{n}-\boldsymbol{\tau}_{n} \cdot \mathbf{v}_{n}\right]=\rho_{n} \mathbf{f} \cdot \mathbf{v}_{n},
\end{gathered}
$$

where $\mathbf{v}_{n}$ is the neutral gas velocity vector, $\epsilon_{n}=$ $p_{n} /\left(\rho_{n}\left(\gamma_{n}-1\right)\right)+\left|\mathbf{v}_{n}\right|^{2} / 2$ is the specific total energy, $\boldsymbol{\tau}_{n}$ is a second order tensor or dyadic quantity representing the neutral gas viscous stresses with elements

$$
\tau_{n_{i j}}=\mu_{n}\left(\frac{\partial v_{n_{i}}}{\partial x_{j}}+\frac{\partial v_{n_{j}}}{\partial x_{i}}-\delta_{i j} \frac{2}{3} \boldsymbol{\nabla} \cdot \mathbf{v}_{n}\right),
$$

I is the unit tensor, $\mathbf{q}_{n}$ is the heat flux vector given by Fourier's law

$$
\mathbf{q}_{n}=-\kappa_{n} \boldsymbol{\nabla} T_{n},
$$

$\mu_{n}$ is the dynamic viscosity, $\kappa_{n}$ is the thermal conductivity, and $\gamma_{n}$ is the specific heat ratio for the neutral gas. Sutherland's law is used to prescribe the viscosity. The vector quantity $\mathbf{f}$ is the external body force per unit mass acting on the neutral gas.

For a two-dimensional axisymmetric coordinate system, the Navier-Stokes equations with $\mathbf{f}=0$ can be re-expressed as:

$$
\frac{\partial \mathbf{U}_{n}}{\partial t}+\frac{\partial \mathbf{F}_{n}}{\partial z}+\frac{\partial \mathbf{G}_{n}}{\partial r}=\frac{\partial \mathbf{F}_{\mathbf{v} n}}{\partial z}+\frac{\partial \mathbf{G}_{\mathbf{v} n}}{\partial r}+\mathbf{S}_{\mathbf{a} n},
$$

where $\mathbf{U}_{n}$ is the neutral gas conserved variable solution vector given by

$$
\mathbf{U}_{n}=\left[\begin{array}{llll}
\rho_{n}, & \rho_{n} u_{n}, & \rho_{n} v_{n}, & \rho_{n} \epsilon_{n}
\end{array}\right]^{T},
$$

$\mathbf{F}_{n}$ and $\mathbf{G}_{n}$ are the axial- and radial-direction inviscid flux vectors given by

$$
\mathbf{F}_{n}=\left[\begin{array}{c}
\rho_{n} u_{n} \\
\rho_{n} u_{n}^{2}+p_{n} \\
\rho_{n} u_{n} v_{n} \\
u_{n}\left(\rho_{n} \epsilon_{n}+p_{n}\right)
\end{array}\right], \mathbf{F}_{n}=\left[\begin{array}{c}
\rho_{n} v_{n} \\
\rho_{n} u_{n} v_{n} \\
\rho_{n} v_{n}^{2}+p_{n} \\
v_{n}\left(\rho_{n} \epsilon_{n}+p_{n}\right)
\end{array}\right],
$$

and $\mathbf{F}_{\mathbf{v} n}$ and $\mathbf{G}_{\mathbf{v} n}$ are the axial- and radial-direction viscous flux vectors given by

$$
\begin{gathered}
\mathbf{F}_{\mathbf{v} n}=\left[\begin{array}{c}
0 \\
\tau_{n_{z z}} \\
\tau_{n_{r z}} \\
u_{n} \tau_{n_{z z}}+v_{n} \tau_{n_{r z}}-q_{n_{z}}
\end{array}\right], \\
0 \\
\mathbf{G}_{\mathbf{v} n}=\left[\begin{array}{c}
\tau_{n_{r z}} \\
\tau_{n_{r r}} \\
u_{n} \tau_{n_{r z}}+v_{n} \tau_{n_{z z}}-q_{n_{r}}
\end{array}\right] .
\end{gathered}
$$


The vector $\mathbf{S}_{\mathbf{a} n}$ contains source and viscous flux terms associated with the axisymmetric geometry and has the form

$$
\mathbf{S}_{\mathbf{a} n}=\frac{1}{r}\left[\begin{array}{c}
-\rho_{n} v_{n} \\
-\rho_{n} u_{n} v_{n}+\tau_{n_{r z}} \\
-\rho_{n} v_{n}^{2}+\tau_{n_{r r}}-\tau_{n_{\theta \theta}} \\
-v_{n}\left(\rho_{n} \epsilon_{n}+p_{n}\right)+u_{n} \tau_{n_{r z}}+v_{n} \tau_{n_{r r}}-q_{n_{r}}
\end{array}\right]
$$

In this case, the components of the viscous stress tensor are given by

$$
\begin{gathered}
\tau_{n_{z z}}=\frac{4}{3} \mu_{n} \frac{\partial u_{n}}{\partial z}-\frac{2}{3} \mu_{n} \frac{\partial v_{n}}{\partial r}-\frac{2}{3} \mu_{n} \frac{v_{n}}{r}, \\
\tau_{n_{r r}}=\frac{4}{3} \mu_{n} \frac{\partial v_{n}}{\partial r}-\frac{2}{3} \mu_{n} \frac{\partial u_{n}}{\partial z}-\frac{2}{3} \mu_{n} \frac{v_{n}}{r}, \\
\tau_{n_{\theta \theta}}=\frac{4}{3} \mu_{n} \frac{v_{n}}{r}-\frac{2}{3} \mu_{n} \frac{\partial u_{n}}{\partial z}-\frac{2}{3} \mu_{n} \frac{\partial v_{n}}{\partial r}, \\
\tau_{n_{r z}}=\mu_{n}\left(\frac{\partial u_{n}}{\partial r}+\frac{\partial v_{n}}{\partial z}\right),
\end{gathered}
$$

and the axial and radial components of the heat flux vector are

$$
q_{n_{z}}=-\kappa_{n} \frac{\partial T_{n}}{\partial z}, \quad q_{n_{r}}=-\kappa_{n} \frac{\partial T_{n}}{\partial r} .
$$

Equation (18) is used here to model the high-speed flow of the neutral gas.

\section{Laplace Equation for Electric Potential}

This study is concerned with the influence of steady (time-invariant) electric fields on ion transport through a high-speed neutral gas. Assuming that the net charge density in the fluid volume of interest is negligible (i.e., although there is an excess of charge due to the lack electrons, ion space charge effects can be neglected because of the low ion number densities), the stationary applied electric field, E, satisfies Gauss's law $\boldsymbol{\nabla} \cdot \mathbf{E}=0$. By expressing the electric field in terms of an electric potential, $V$, such that $\mathbf{E}=-\nabla V$, the electric field can be determined by solving a Laplace equation for the electric potential

$$
\nabla^{2} V=0
$$

subject to appropriate boundary conditions. For two-dimensional axisymmetric coordinate the Laplace equation has the form

$$
\frac{\partial^{2} V}{\partial z^{2}}+\frac{\partial^{2} V}{\partial r^{2}}+\frac{1}{r} \frac{\partial V}{\partial r}=0
$$

The solution of this scalar equation provides a description of the applied steady-state electric field.

\section{Numerical Solution Procedure}

\section{Solution of Ion Transport Equations}

A parallel explicit higher-order Godunov-type finitevolume scheme is used to solve the five-moment ion transport equations given by Eq. (6) on multi-block quadrilateral mesh. The proposed scheme determines the ion motion given a precomputed neutral gas flow field and electric potential. Upwind finite-volume schemes were originally introduced by Godunov. ${ }^{16}$ Application and further development of these schemes for the equations of gas dynamics has been well documented in literature. ${ }^{17-30}$ Solution procedures for the neutral gas flow field and electric field are discussed in subsequent subsections.

In the present finite-volume approach, the governing equations are integrated over quadrilateral cells of a structured multi-block quadrilateral mesh. The finitevolume formulation applied to cell $j$ is given by

$\frac{d \mathbf{U}_{i_{j}}}{d t}=-\frac{1}{A_{j}} \sum_{f a c e s, k} \overrightarrow{\mathbf{F}}_{i_{j k}} \cdot \overrightarrow{\mathbf{n}}_{j k} \Delta \ell_{j k}+\mathbf{S}_{\mathbf{a} i_{j}}+\mathbf{S}_{\mathbf{e} i_{j}}+\mathbf{S}_{\mathbf{c} i_{j}}$,

where $\overrightarrow{\mathbf{F}}_{i}=\left(\mathbf{F}_{i}, \mathbf{G}_{i}\right), A_{j}$ is the area of cell $j$, and $\Delta \ell_{j k}$ and $\overrightarrow{\mathbf{n}}_{j k}$ are the length of the cell face $k$ and unit vector normal to the cell face or edge, respectively. The numerical fluxes at the faces of each cell are determined from the solution of a Riemann problem. Given the left and right solution states, $\mathbf{U}_{i_{l}}$ and $\mathbf{U}_{i_{r}}$, at the cell interfaces, the numerical flux is given by

$$
\overrightarrow{\mathbf{F}}_{i} \cdot \overrightarrow{\mathbf{n}}=\mathcal{F}_{i}\left(\mathbf{U}_{i_{l}}, \mathbf{U}_{i_{r}}, \mathbf{n}\right),
$$

where the numerical flux $\mathcal{F}_{i}$ is evaluated by solving a Riemann problem in a direction defined by the normal to the face with initial data $\mathbf{U}_{l}$ and $\mathbf{U}_{r}$. The left and right solution states are determined using the leastsquares piece-wise limited linear solution reconstruction procedure of Barth. ${ }^{31}$ The modified limiter of Venkatakrishnan $^{32}$ has also been implemented. In the present algorithm, both exact and approximate Riemann solvers can be used to solve the Riemann problem. The Roe linearized Riemann solver, ${ }^{22}$ HLLE-type flux function of Linde, ${ }^{33}$ the HLLC flux function, ${ }^{34}$ and the exact Riemann solver of Gottlieb and Groth ${ }^{35}$ have all been implemented and may be used. For timeaccurate calculations, predictor-corrector and fourth order Runge-Kutta time-marching methods are used to integrate the set of ordinary differential equation that result from this spatial discretization of the governing equations. The optimally-smoothing multistage schemes developed by van Leer et $a l^{36}$ are adopted for steady-state calculations. To cope with numerical stiffness, a semi-implicit treatment is used in the temporal discretization of the source terms associated with axisymmetric geometry, electric field, and ion-neutral collisions. 


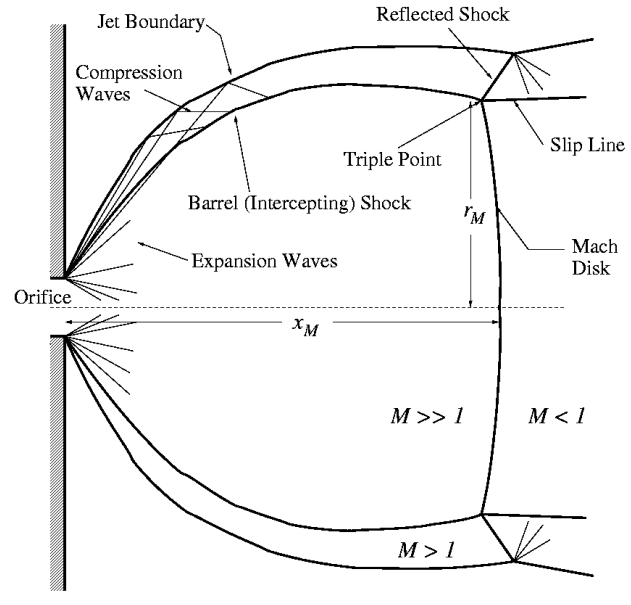

Fig. 1 Schematic diagram of free jet flow shock structure.

Following the approach developed by Groth et al. for computational magnetohydrodynamics, ${ }^{37,38}$ a flexible block-based hierarchical data structure is used to maintain the connectivity of the quadrilateral solution blocks in the multi-block mesh and facilitate automatic solution-directed mesh adaptation according to physics-based refinement criteria. This data structure also lends itself naturally to domain decomposition and thereby enables efficient and scalable implementations of the algorithm on distributed-memory multi-processor architectures.

\section{Solution of Navier-Stokes Equations for Neutral Flow}

A commercial computational fluid dynamics flow solver $(\mathrm{CFD}++$, developed by Metacomp Technologies) is used to predict the high-speed expanding neutral gas flows. This flow solver employs an upwind total variation diminishing (TVD) finite-volume spatial discretization scheme in conjunction with a multigrid accelerated implicit time-marching procedure to solve the compressible axisymmetric form of the Navier-Stokes equations on unstructured triangular mesh. ${ }^{39,40}$ A V-cycle and Gauss-Seidel smoother are employed in the multigrid convergence acceleration strategy. Clustering of the cells in the computational mesh may be used to help to capture the important features of the complex neutral flows. The commercial solver also has parallel processing capabilities. The parallel implementation of the neutral gas solver has been developed using the MPI library ${ }^{41}$ for performing inter-processor communications and the METIS graph partitioning software for performing a domain decomposition of the computational mesh. ${ }^{42}$ The neutral flow solution is independent of the ion solution and can be obtained in a separate calculation. Linear interpolation is used to transfer the neutral flow solution from the unstructured triangular mesh used in the so-

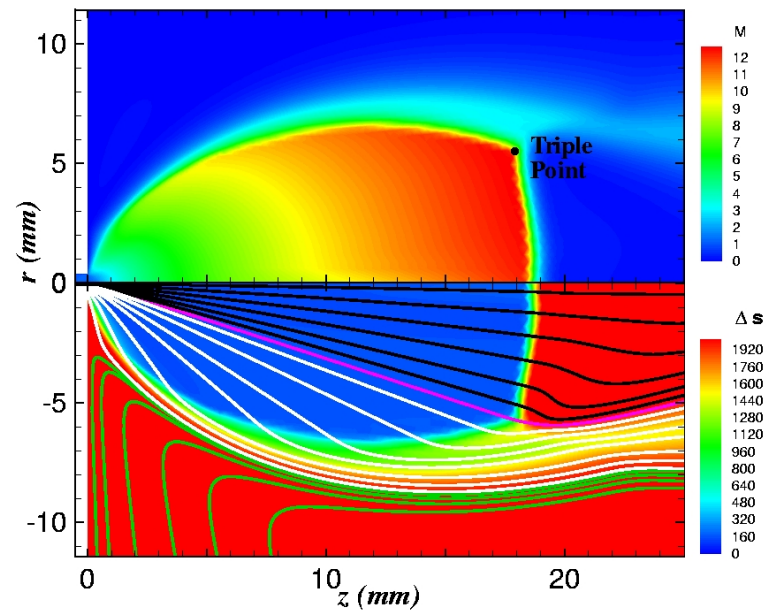

Fig. 2 Computed neutral gas streamlines and distributions of Mach number, $M_{n}$, and entropy change, $\Delta s_{n}$, for underexpanded free-jet flow with an orifice diameter of $d_{\circ}=0.75$ $m m$ and pressure ratio $p_{\circ} / p_{b}=1520$.

lution of the neutral flow equations to the multi-block quadrilateral mesh used by the finite-volume scheme for the ion transport equations.

\section{Solution of Electric Potential}

An iterative solution procedure is used to solve Eq. (30) for the electric potential, $V$, and the electric field is then calculated in terms of the gradient of the potential, $\mathbf{E}=-\boldsymbol{\nabla} V$. Like the neutral flow solution, the electric field is not dependent on the predicted ion solution and can also be determined in a separate independent computation.

\section{Results and Discussion}

The mathematical formulation and solution algorithm described in previous paragraphs are used to perform the numerical simulations of the ion transport. A detailed discussion of the results of the numerical simulations of ion motion for high-speed, underexpanded, axisymmetric, jet flows are given. The simulations investigate both free jets and jet flows with flow gas skimmers. Some numerical results for axisymmetric free jet flows are first described.

\section{Axisymmetric Free-Jet Flow}

When a high pressure gas is exhausted through an orifice into a stagnant low-pressure chamber, the gas rapidly expands and results in the formation of an under-expanded free jet. ${ }^{43-45}$ A schematic diagram of the under-expanded free jet is shown in Figure 1 . At the orifice, the flow is for the most part supersonic. The upstream high pressure gas is therefore unaffected by the downstream flow conditions and the jet structure must adjust to match the back pressure in the 


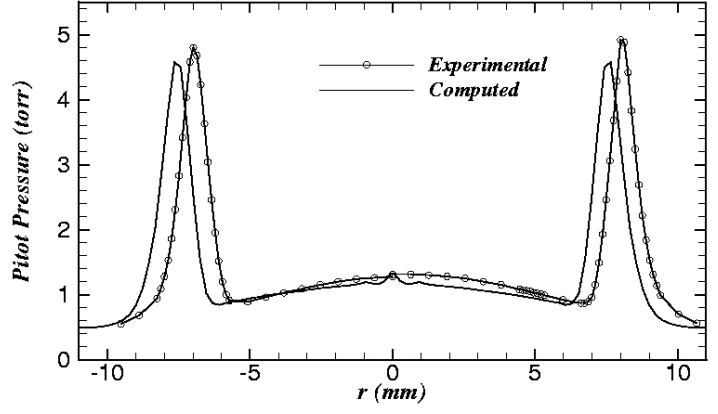

Fig. 3 Comparison of computed and measured radial pitot probe neutral gas pressure profiles $13.97 \mathrm{~mm}$ downstream from the orifice for under-expanded free-jet flow with an orifice diameter $d_{\circ}=0.75 \mathrm{~mm}$ and pressure ratio $p_{\circ} / p_{b}=$ 1520 .

low-pressure chamber. The resulting free-jet flow is quite complex and characterized by thin non-isentropic regions (shocks) with large gradients in the properties of the gas. Key features of an under-expanded free jet include the: (i) barrel (intercepting) shock; (ii) jet boundary; (iii) triple point; (iv) Mach disk; and (v) reflected shock.

The numerical solution of an under-expanded freejet flow of a neutral gas consisting of diatomic nitrogen obtained using using a 41,000-cell unstructured triangular grid is shown in Figure 2. For this case, the orifice diameter, $d_{\circ}$, is $0.75 \mathrm{~mm}$, the upstream pressure, $p_{\circ}$, and temperature, $T_{\circ}$, are 760 torr and 288 $\mathrm{K}$, and the downstream pressure, $p_{b}$, is 0.5 torr, respectively. The figure depicts the predicted neutral flow streamlines and computed distributions of Mach number $\mathrm{M}_{n}=|\mathbf{v}|_{n} / a_{n}$, and entropy change $\Delta s_{n}=$ $\left(R_{n} /\left(\gamma_{n}-1\right)\right) \ln \left(\left(p_{n} / p_{\circ}\right) /\left(\rho_{n} / \rho_{\circ}\right)_{n}^{\gamma}\right)$, where $a_{n}$ is the sound speed of the neutral gas. The black coloured streamlines are associated with neutral gas flow that passes through the Mach disk whereas the white coloured stream traces are associated with flow that passes through a region above of the triple point via the intercepting shock. The purple coloured streamline marks the boundary between flow passing through the Mach disk and flow passing above the triple point. The green coloured stream traces reflect the viscous entrainment of the low-pressure reservoir gas into the free jet flow that occurs in the mixing layer at the jet boundary. The flow is highly expanded downstream of the orifice. The pressure decreases by five orders in magnitude in the free jet and then increases abruptly again following the Mach disk to match the background pressure. The density and temperature also decrease and the flow speed increases monotonically. The Mach number exceeds 12 in the core region of jet. The intercepting shock, jet boundary, triple

\begin{tabular}{||l||c|c|c|c||}
\hline \hline Ion & $A$ & $B$ & $C$ & $D$ \\
\hline \hline Mass (a.m.u) & 105 & 228 & 609 & 16951 \\
\hline Charge & +1 & +1 & +1 & +9 \\
\hline$\sigma_{i}\left(10^{-20} m^{2}\right)$ & 105 & 150 & 280 & 2560 \\
\hline$\gamma_{i}$ & 1.2 & 1.1 & 1.1 & 1.05 \\
\hline \hline IF2 (\%) & 94 & 86 & 53 & 39 \\
\hline IF3 (\%) & 59 & 68 & 36 & 38 \\
\hline
\end{tabular}

Table 1 Physical properties of ions and ion flux through skimmer (IF2) and downstream of the skimmer (IF3) as a percentage of the reference value through the orifice (IF1) for an orifice diameter of $d_{\circ}=0.25 \mathrm{~mm}$. Refer to Figure 6 for the locations of station IF1, IF2, and IF3.

point, Mach disk, and reflected shock are all quite evident in the the predicted entropy and Mach number distributions and appear to be well resolved.

Results of a comparison between measured and predicted neutral gas pitot pressures for this free-jet flow are given in Figure 3. The figure depicts computed and measured radial profiles of the pressure at a distance of $13.97 \mathrm{~mm}$ downstream of the orifice. This corresponds to a location upstream of the Mach disk. Although, the experimental data is not quite symmetrical about $r=0$ due to slight asymmetries in the experimental jet and uncertainties in the actual location of the axis of symmetry, the two profiles both have the same general topology. There is a pressure plateau in the core region of the jet, followed by a sharp increase in the stagnation pressure across the barrel shock, and finally a decrease in pressure to match the background value of 0.5 torr. Comparisons at other radial and axial stations yield similar agreement. ${ }^{46}$ Although, there is some disagreement between measured and predicted values of the pitot pressure close to the orifice (this difference is most likely due to probe interference effects), overall the agreement between the experimental data and numerical results is very good, providing strong support for the validity and reliability of the neutral gas calculations. In particular, the excellent agreement in the pressure profiles would imply that the boundaries and extent of the free jet are well predicted by the neutral gas flow solver.

As a first step to understanding ion transport processes in high-speed jets, the transport of ion particles through the preceding under-expanded free jet flow was studied.

The four positively-charged ion particles described in Table 1 correspond respectively to: A (a generic low mass-to-charge ion); B (dodecyltrimethyl ammonium, $\mathrm{C}_{15} \mathrm{H}_{34} \mathrm{~N}$ ); $\mathrm{C}$ (reserpine protonated molecule) and $\mathrm{D}$ (myoglobin - a protein). In particular, the transport of singly-charged ion particles (ion B from Table 1),

6 


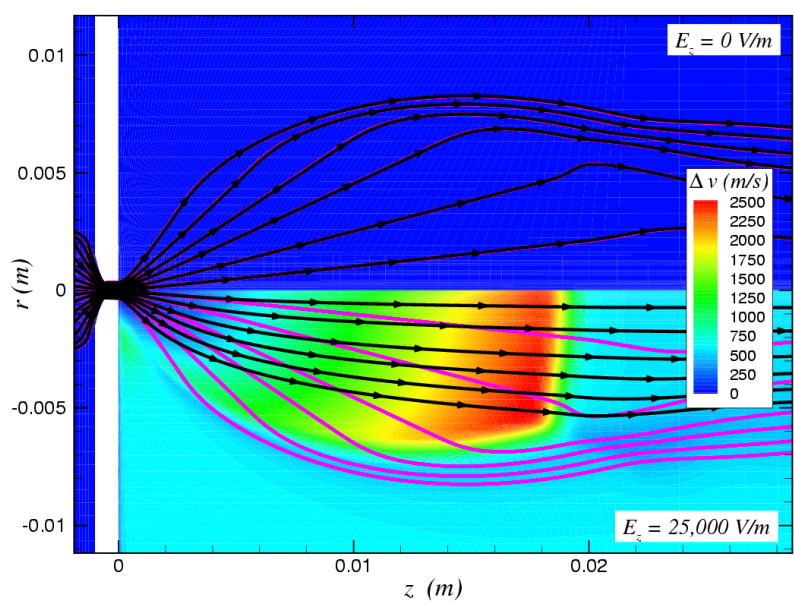

Fig. 4 Computed ion particle (black) and neutral gas (purple) streamlines and distributions of the difference between the ion and neutral gas velocities $\Delta v=\left|\mathbf{v}_{i}-\mathbf{v}_{n}\right|$, for underexpanded free-jet flow with an orifice diameter of $d_{\circ}=0.75$ $m m$ and pressure ratio $p_{\circ} / p_{b}=1520$. Results are shown for two values of the axial component of the electric field: $E_{z}=0 \mathrm{~V} / \mathrm{m}$ (upper-half plane) and $E_{z}=25,000 \mathrm{~V} / \mathrm{m}$ (lower-half plane).

is first considered with a molecular weight of $228 \mathrm{amu}$ specific heat ratio $\gamma_{i}=1.2$, and a constant cross section of $\sigma_{i n}=150(10)^{-20} \mathrm{~m}^{2}$ for collisions with neutral nitrogen particles. The ions upstream of the orifice were assumed to be in equilibrium with the neutral gas and the upstream number density of the ions was taken to be $n_{i}=(10)^{14} \mathrm{~m}^{-3}$. Constant axial electric fields of varying magnitude were applied and numerical solutions were obtained using the parallel finite-volume method described above. A total of 13 solutions blocks and 24,500 quadrilateral computational cells were used in the computations.

Figures 4 and 5 show the numerical results for the ion transport in the case of an under-expanded free jet. The computed ion and neutral gas streamlines, electric field lines, and distributions of the differences between the ion and neutral gas velocities and temperatures are shown. In the absence of an electric field $\left(E_{z}=0 \mathrm{~V} / \mathrm{m}\right)$, it can be seen that the ion-neutral collision frequency is sufficient to maintain equilibrium conditions between ions and neutral particles such that $\mathbf{v}_{i}=\mathbf{v}_{n}$ and $T_{i}=$ $T_{n}$. When the electric field becomes sufficiently strong $\left(E_{z}=25,000 \mathrm{~V} / \mathrm{m}\right)$, the electric forces then dominate the ion motion in the high-speed regions of the free jet flow downstream of the orifice with low neutral gas density. The ion particles undergo a rapid acceleration by the electric field and achieve velocities in excess of $2.5 \mathrm{~km} / \mathrm{s}$ above those of the neutrals. At the same time the Joule heating and drag forces due to collisions with the neutrals produce significant heating of the ions.

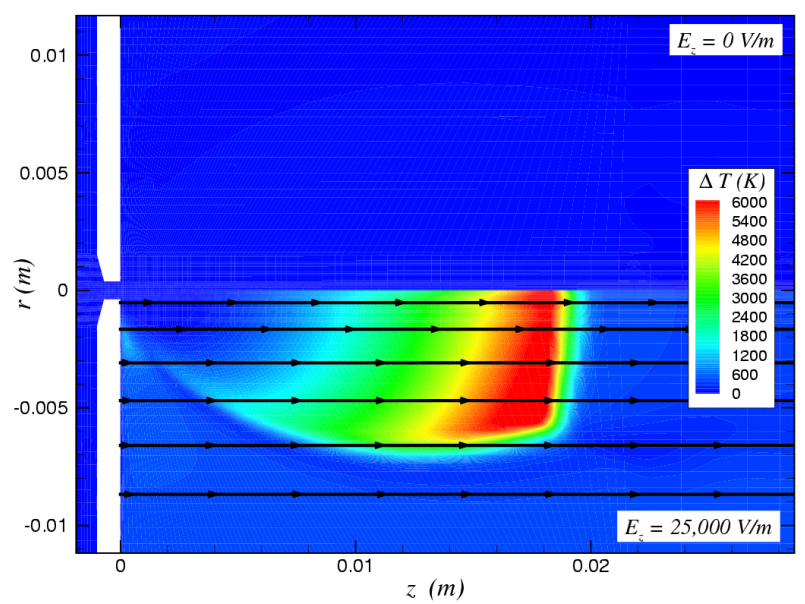

Fig. 5 Computed field lines of the applied electric potential and distributions of the difference between the ion and neutral gas temperatures $\Delta T=T_{i}-T_{n}$, for under-expanded free-jet flow with an orifice diameter of $d_{\circ}=0.75 \mathrm{~mm}$ and pressure ratio $p_{\circ} / p_{b}=1520$. Results are shown for two values of the axial component of the electric field: $E_{z}=0$ $\mathrm{V} / \mathrm{m}$ (upper-half plane) and $E_{z}=25,000 \mathrm{~V} / \mathrm{m}$ (lower-half plane).

Temperatures in excess of $7,000 \mathrm{~K}$ in this particular example are predicted.

\section{Axisymmetric Skimmer Flows}

Having explored features of ion transport for freejet flows, jet flows with neutral gas skimmers are now considered. Gas skimmers, as shown in Figure 6, are introduced in the interface regions of mass spectrometer systems to reduce the flux of neutral gas to the ion optics and to focus ions that are being transported by the neutral flow. Ion transport is studied herein for supersonic jets emanating from orifices of diameters $d_{\circ}=0.75$ and $0.25 \mathrm{~mm}$ with an axisymmetric conicalshaped skimmer having a skimmer orifice diameter of $d_{s}=2.5 \mathrm{~mm}$ and a cone angle of $\alpha_{s}=60^{\circ}$. The transport of ion particles is considered for several different ions with varying mass-to-charge ratios and the influences of the applied electric field and neutral particles collisions are assessed. Table 1 provides a summary of the ions considered. For these cases, differential pumping is used such that the back pressure between the orifice and the skimmer, $p_{b}$, is maintained at 1 torr and the back pressure downstream of the skimmer, $p_{s}$, is lower and has a value of 0.005 torr. In addition, the applied electric is relatively strong: $80 \mathrm{~V}$ is applied to the orifice while the gas skimmer is grounded, yielding values in excess of $(E=70,000 \mathrm{~V} / \mathrm{m})$ near the tip of the skimmer.

The overall structure of the flow with a gas skimmer is very different from that of the free jet. The presence 


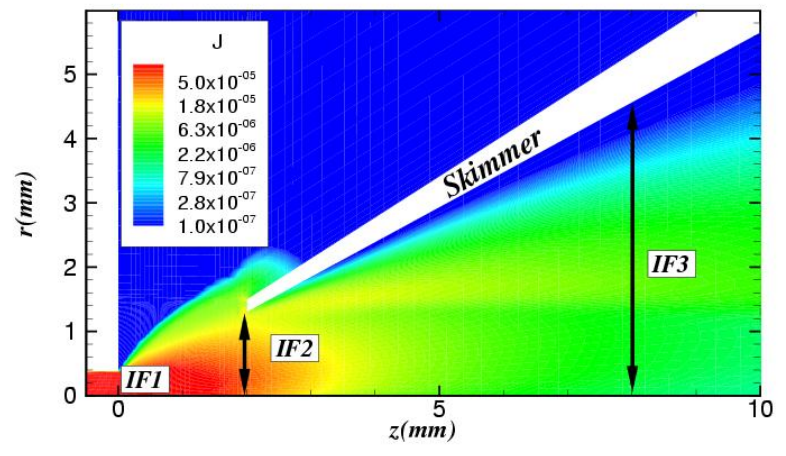

Fig. 6 Computed distributions of the current density, $J=$ $|\mathbf{J}|=Q_{i} n_{i}\left|\mathbf{v}_{i}\right|\left(A / m^{2}\right)$, for Ion $B$ in an under-expanded jet flow with orifice diameter of $d_{\circ}=0.75 \mathrm{~mm}$. Stations IF1, IF2, and IF3 refer to the axial positions where the ion flux tabulated in Table 1 has been evaluated.

of the skimmer disrupts and prevents the jet of from fully expanding. As a consequence, a Mach disk does not form. Unlike the free jet case, the neutral gas remains highly supersonic in the core of the jet far downstream of the orifice and skimmer. The skimmer not only confines the jet expansion process but also acts to divide the neutral flow, diverting a significant portion of the neutral particles. The flow structure of the neutral gas in the presence of skimmers is discussed in detail in the recent work of Jugroot et al. ${ }^{46}$

Figure 6 shows the numerical simulation of ion transport for an ion with a mass-to-charge ratio of 228 (ion B) for the larger diameter jet $\left(d_{\circ}=0.75\right.$ $\mathrm{mm})$. The computed distribution of the current density, $J=|\mathbf{J}|=Q_{i} n_{i}\left|\mathbf{v}_{i}\right|$, for ion B indicates that most of the ions go through the skimmer as a result of combined effects of entrainment by the neutral supersonic gas and the applied electric field. Figure 7 shows additional predictions of ion transport for the smaller jet orifice with an orifice diameter of $0.25 \mathrm{~mm}$. The computed ion and neutral gas streamlines and distribution of ion velocity are shown for both ion $\mathrm{B}$ and ion $\mathrm{D}$. Ion $\mathrm{D}$ has a much larger mass-to-charge ratio of 1884 .

For ion B, it can be observed that the neutral gas is partially diverted by the skimmer whereas all the ion streamlines go through the skimmer. At the exit of the orifice and in its close vicinity, the ions follow the neutral gas, but as they approach the skimmer region, the ion trajectories clearly deviate from those of the neutrals due to the strong imposed electric field. The applied electric field is non-uniform as a result of the point-to-plane type configuration and dominates the ion motion in the high-speed regions of the jet flow downstream of the orifice. In this region the neutral gas becomes very dilute and the governing character of the electric field grows as its magnitude rapidly increases. The ion B particles are strongly accelerated by the electric field and achieve much high velocities

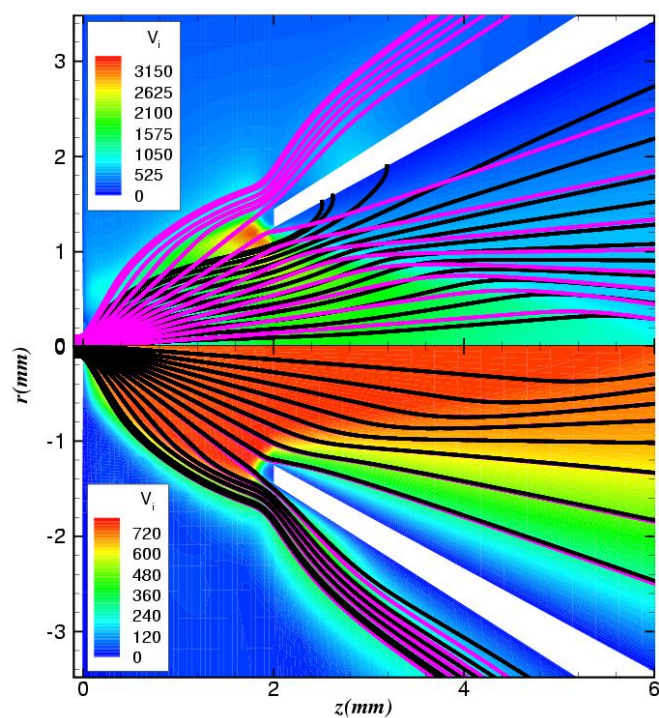

Fig. 7 Computed ion particle (black) and neutral gas (purple) streamlines and distributions of the ion velocity $(\mathrm{m} / \mathrm{s})$ for under-expanded jet flow with an orifice diameter of $d_{\circ}=0.25 \mathrm{~mm}$. Results are shown for two ions: the upper-half plane corresponds to ion $B$ and the lower-half plane to ion $D$.

than the neutrals as depicted in Figure 7. Joule heating and drag forces due to collisions with the neutrals produce heating of the ions near the tip of the skimmer and temperatures exceeding $5,000 \mathrm{~K}$ are observed as shown in Figure 9. Such high temperatures observed for ion B would probably initiate ion breakdown at the tip of the skimmer. It should be further noted that the neutral-to-ion pressure ratio in the core of the jet flow is about $(10)^{10}$, hence justifying the indirect coupling adopted herein for predicting the ion and neutral transport.

The predicted ion motions for the other ions of varying mass-to-charge ratios (ions A, C, and D) are in general the same as that for ion $\mathrm{B}$ in the vicinity just downstream of the jet orifice; however, as the electric field grows in strength and the neutral gas becomes more dilute near the skimmer tip, the differences in the transport of the ions of varying mass-to-charge ratios is becomes readily apparent. The ions exhibit a clear tendency to follow the diverging electric field lines. The extent of this phenomenon is directly dependant on the mass of the ion and its charge. The lowest mass ion A is most influenced by the electric field and follows closely the field lines whereas ion $\mathrm{D}$ is almost unaffected by the electric field due to its high mass as seen in Figures 7 and 8. The tendency for the lower mass ions to follow the electric field has a direct impact on the ion currents (mass flux) through the skimmer. The computed ion fluxes ratios for ions A, B, C, and $\mathrm{D}$ at the skimmer (station IF2) and at station IF3 6 $\mathrm{mm}$ downstream of the skimmer, normalized to the 


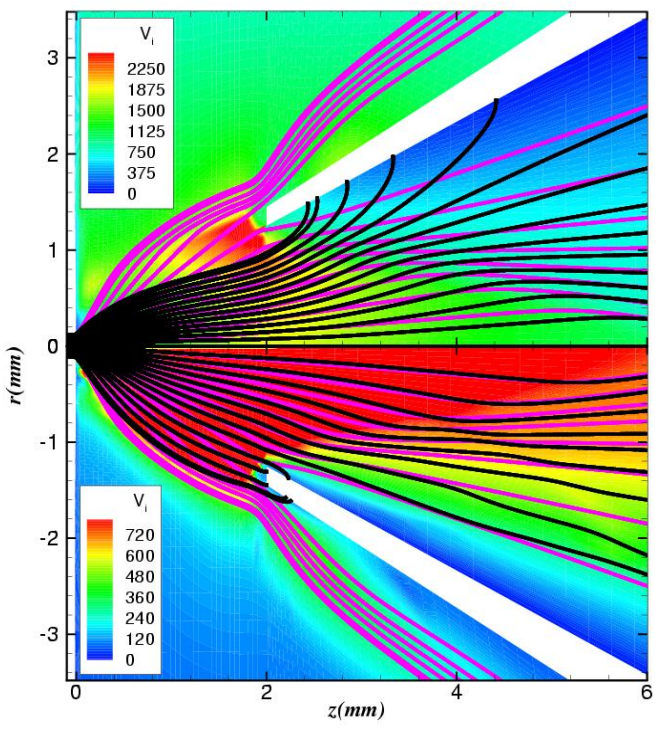

Fig. 8 Computed ion particle (black) and neutral gas (purple) streamlines and distributions of the ion velocity $(\mathrm{m} / \mathrm{s})$ for under-expanded jet flow with an orifice diameter of $d_{\circ}=0.25 \mathrm{~mm}$. Results are shown for two ions: the upper-half plane corresponds to ion $A$ and the lower-half plane to ion $C$.

reference value determined at the exit of the jet orifice (station IF1) are summarized in Table 1. The electric field causes a greater number of the lower mass ions to pass through the skimmer ( $94 \%$ of ion A particles pass through the skimmer orifice as compared to $39 \%$ for ion D). Interestingly enough, the lower mass ions are also more readily induced by the diverging electric field to impact the skimmer downstream of the skimmer orifice thereby reducing the effective ion mass flux through the skimmer (only $59 \%$ of ion A particles pass through the skimmer at station IF3 whereas virtually all ion $\mathrm{D}$ particles that pass through the skimmer orifice also pass through the skimmer at station IF3).

Beyond the skimmer tip region, where the electric field gradually diminishes, all ions tend to follow the neutral gas trajectory, characterized by a flow deflection produced by the skimmer leading edge conicalshaped shock that subsequently reflects from the axis of symmetry. ${ }^{46}$ In the absence of an applied electric field $(E=|\mathbf{E}|=0 \mathrm{~V} / \mathrm{m})$, the ion-neutral collision frequency is sufficient to maintain equilibrium conditions between ions and neutral particles such that $\mathbf{v}_{i}=\mathbf{v}_{n}$ and $T_{i}=T_{n}$. Thus differences between the ion and neutral trajectories are negligible for all of the ions considered here when the electric field is absent.

A better understanding of the ion transport can be obtained by considering the relative effects of ionneutral collision processes on the ion motion as compared to the effects of the applied electric field. The ratio of 1-norm of the momentum source terms as-

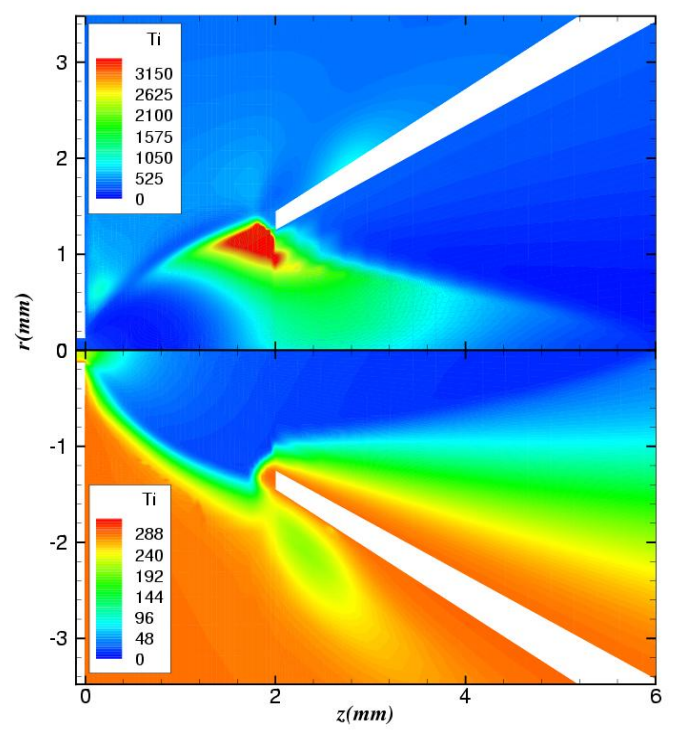

Fig. 9 Computed distribution of ion temperature (K) for under-expanded jet flow with an orifice diameter of $d_{\circ}=$ $0.25 \mathrm{~mm}$. Results are shown for two ions: the upper-half plane corresponds to ion $B$ and the lower-half plane to ion D.

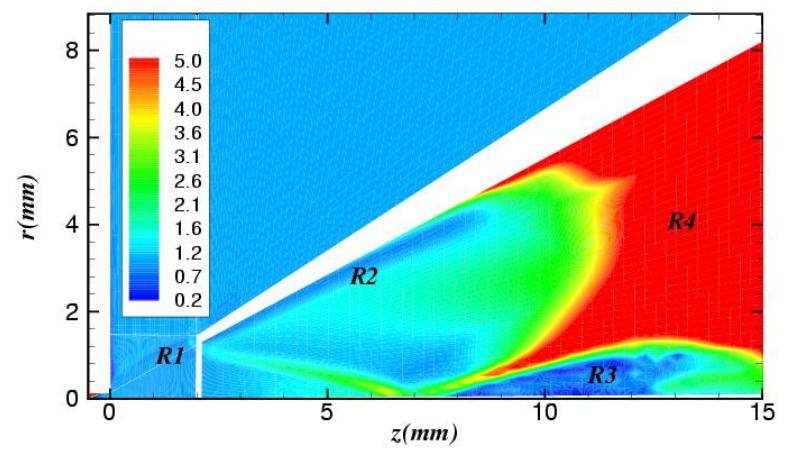

Fig. 10 Computed distribution of the ratio of the 1-norm of the momentum source terms due to ion-neutral collisions to the 1-norm of the moment sources terms for the electric field as given in Eqs. (10) and (11)), for Ion A in an underexpanded jet flow with orifice diameter of $d_{\circ}=0.25 \mathrm{~mm}$.

sociated with ion-neutral collisions (both axial and radial momentum components) to the 1 -norm of the momentum source terms due to the electric field can be evaluated. Refer to Eqs. (10) and (11). This ratio provides an indication of the relative importance of the neutral drag due to collisional processes as compared to the ion acceleration produced by the imposed electric field. Computed values of this ratio are depicted in Figure 10 for Ion A in an under-expanded jet flow with orifice diameter of $d_{\circ}=0.25 \mathrm{~mm}$. It is interesting to observe that different regions of the simulated flow field are either collisionally regulated or regulated by the electric field, while still other regions of the flow exhibit an equilibrium between these two competing 
effects. Near the tip of the skimmer (Region R1), the electric field is only slightly dominant, in spite of the fact that the electric field is very strong in this region. This is because the neutral gas density rises sharply in the vicinity of the skimmer bow shock. However, in region $\mathrm{R} 2$, it is clear that the electric field effect predominates. The electric field effect is again more dominant in region R3, but not due to a strong applied electric field but rather as a result of the neutral density rising again after the skimmer bow shock reflects from the axis of symmetry. Near equilibrium conditions for the ion and neutral gas velocities are attained in this region. Further downstream in region $\mathrm{R} 4$, collisional effects exert more influence on the ion motions as the electric field becomes negligible.

\section{Concluding Remarks}

The transport of free ions through highly underexpanded jet flows of neutral gases and in the presence of applied electric fields has been investigated using continuum-based numerical simulations. A fivemoment mathematical model and parallel multi-block numerical solution procedure has been developed and described for predicting the ion transport. The model incorporates the effects of ion-neutral collision processes and is used in conjunction with a Navier-Stokes flow solver for the neutral gas to examine the key features of the ion motion. The influences of the neutral gas flow, electric field, and flow field geometry on ion mobility has been demonstrated for typical flow conditions and geometries found in the interface regions of mass spectrometer systems. The combined effect of the applied electric field and neutral collision processes with the diluted background gas results in a tendency for ion focusing toward the axis, with the overall efficiency of the focusing being governed by the mass-to-charge ratio. Future work will involve extending the modelling to three-dimensional flow geometries and investigating the application of high-order moment models to describe non-equilibrium ion transport phenomena.

\section{Acknowledgments}

This research was supported by the Natural Sciences and Engineering Research Council (NSERC CRDPJ 254794-01). Funding for the parallel computing facility used to perform the computations described herein was obtained from the Canadian Foundation for Innovation and Ontario Innovation Trust (CFI Project No. 2169). The authors are very grateful to these funding agencies for this support.

\section{References}

${ }^{1}$ Meyyappan, M., editor, Computational Modeling in Semiconductor Processing, Artech House, Boston, 1995.
${ }^{2}$ Meyyappan, M., "Plasma Process Modeling", In Meyyappan, M., editor, Computational Modeling in Semiconductor Processing, pages 231-323. Artech House, Boston, 1995.

${ }^{3}$ Jugroot, M., Bayle, P., Yousfi, M., and Eichwald, O., "Self-Consistent Modelling of Charged and Neutral Particle Dynamics in Short-Gap Helium and Hydrogen Discharges", J. Phys. D: Appl. Phys., Vol. 32, No. 2, pp. 106-120, 1999.

${ }^{4}$ Brophy, J. R., and Noca, M., "Electric Propulsion for Solar System Exploration", J. Propulsion Power, Vol. 14, No. 5, pp. 700-707, 1998.

${ }^{5}$ Martinez-Sanchez, M., and Pollard, J. E., "Spacecraft Electric Propulsion - An Overview", J. Propulsion Power, Vol. 14, No. 5, pp. 688-699, 1998.

${ }^{6}$ Jugroot, M., and Harvey, J. K., "Simulation of Flows within an Electrostatic Ion Thruster for Space Missions", Aeronaut. J., Vol. 105, No. 1053, pp. 613618, 2001.

${ }^{7}$ Krülle, G., Auweter-Kurtz, M., and Sasoh, A., "Technology and Application Aspects of Applied Field Magnetoplasmadynamic Propulsion", J. Propulsion Power, Vol. 14, No. 5, pp. 754-763, 1998.

${ }^{8}$ Schunk, R. W., and Nagy, A. F., Ionospheres: Physics, Plasma Physics, and Chemistry, Cambridge University Press, New York, 2000.

${ }^{9}$ Cravens, T. E., Physics of Solar System Plasmas, Cambridge University Press, New York, 1997.

${ }^{10}$ Thomas, R., "Recent Developments in LC-MS-MS for the Identification and Measurement of Nanoscale Amounts of Proteins and Peptides", Spectroscopy, Vol. 16, No. 1, pp. 28-37, 2001.

${ }^{11}$ Burgers, J. M., Flow Equations for Composite Gases, Academic Press, New York, 1969.

${ }^{12}$ Barakat, A. R., and Schunk, R. W., "Transport Equations for Multicomponent Anisotropic Space Plasmas: A Review", Plasma Phys., Vol. 24, pp. 389 418, 1982.

${ }^{13}$ Gombosi, T. I., and Rasmussen, C. E., "Transport of Gyration-Dominated Space Plasmas of Thermal Origin 1. Generalized Transport Equations", J. Geophys. Res., Vol. 96, No. A5, pp. 7759-7778, 1991.

${ }^{14}$ Gombosi, T. I., Gaskinetic Theory, Cambridge University Press, Cambridge, 1994.

${ }^{15}$ Bhatnagar, P. L., Gross, E. P., and Krook, M., "A Model for Collision Processes in Gases. I. Small Amplitude Processes in Charged and Neutral OneComponent Systems", Physical Rev., Vol. 94, No. 3, pp. 511-525, 1954.

${ }^{16}$ Godunov, S. K., "Finite-Difference Method for Numerical Computations of Discontinuous Solutions of the Equations of Fluid Dynamics", Mat. Sb., Vol. 47, pp. 271-306, 1959. 
${ }^{17}$ van Leer, B., "Towards the Ultimate Conservative Difference Scheme. I. The Quest of Monotonicity", In Lecture Notes in Physics, volume 18, pages 163-168, New York, 1973. Springer-Verlag.

${ }^{18}$ van Leer, B., "Towards the Ultimate Conservative Difference Scheme. II. Monotonicity and Conservation Combined in a Second Order Scheme", J. Comput. Phys., Vol. 14, pp. 361-370, 1974.

${ }^{19}$ van Leer, B., "Towards the Ultimate Conservative Difference Scheme. III. Upstream-Centered FiniteDifference Schemes for Ideal Compressible Flow", J. Comput. Phys., Vol. 23, pp. 263-275, 1977.

${ }^{20}$ van Leer, B., "Towards the Ultimate Conservative Difference Scheme. IV. A New Approach to Numerical Convection", J. Comput. Phys., Vol. 23, pp. 276-299, 1977.

${ }^{21}$ van Leer, B., "Towards the Ultimate Conservative Difference Scheme. V. A Second-Order Sequel to Godunov's Method", J. Comput. Phys., Vol. 32, pp. 101136, 1979 .

${ }^{22}$ Roe, P. L., "Approximate Riemann Solvers, Parameter Vectors, and Difference Schemes", J. Comput. Phys., Vol. 43, pp. 357-372, 1981.

${ }^{23}$ van Leer, B., "Flux-Vector Splitting for the Euler Equations", In Lecture Notes in Physics, volume 170, page 507, New York, 1982. Springer-Verlag.

${ }^{24}$ Colella, P., and Woodward, P. R., "The Piecewise Parabolic Method (PPM) for Gas-Dynamical Simulations", J. Comput. Phys., Vol. 54, pp. 174-210, 1984.

${ }^{25}$ Harten, A., "High Resolution Schemes for Hyperbolic Conservation Laws", J. Comput. Phys., Vol. 49, pp. 357-393, 1983.

${ }^{26}$ Harten, A., "On a Class of High Resolution TotalVariation-Stable Finite-Difference Schemes", SIAM J. Numer. Anal., Vol. 21, pp. 1-23, 1984.

${ }^{27}$ Osher, S., and Chakravarthy, S. R., "High Resolution Schemes and the Entropy Condition", SIAM J. Numer. Anal., Vol. 21, No. 5, pp. 955-984, 1984.

${ }^{28}$ Roe, P. L., "Generalized Formulation of TVD LaxWendroff Schemes", Report 84-53, ICASE, January 1984.

${ }^{29}$ Yee, H. C., "Construction of Explicit and Implicit Symmetric TVD Schemes and Their Applications", J. Comput. Phys., Vol. 68, pp. 151-179, 1987.

${ }^{30}$ Harten, A., Enquist, B., Osher, S., and Chakravarthy, S. R., "Uniformly High Order Accurate Essentially Non-Oscillatory Schemes, III", J. Comput. Phys., Vol. 71, pp. 231-303, 1987.

${ }^{31}$ Barth, T. J., "Recent Developments in High Order K-Exact Reconstruction on Unstructured Meshes", Paper 93-0668, AIAA, January 1993.

${ }^{32}$ Venkatakrishnan, V., "On the Accuracy of Limiters and Convergence to Steady State Solutions", Paper 93-0880, AIAA, January 1993.
${ }^{33}$ Linde, T. J., A Three-Dimensional Adaptive Multifluid MHD Model of the Heliosphere, PhD thesis, University of Michigan, May 1998.

${ }^{34}$ Toro, E. F., Spruce, M., and Speares, W., "Restoration of the Contact Surface in the HLLRiemann solver", Shock Waves, Vol. 4, No. 1, pp. 2534, 1994.

${ }^{35}$ Gottlieb, J. J., and Groth, C. P. T., "Assessment of Riemann Solvers for Unsteady One-Dimensional Inviscid Flows of Perfect Gases", J. Comput. Phys., Vol. 78, pp. 437-458, 1988.

${ }^{36}$ van Leer, B., Tai, C. H., and Powell, K. G., "Design of Optimally-Smoothing Multi-Stage Schemes for the Euler Equations", Paper 89-1933-CP, AIAA, June 1989.

${ }^{37}$ Groth, C. P. T., Zeeuw, D. L. D., Powell, K. G., Gombosi, T. I., and Stout, Q. F., "A Parallel SolutionAdaptive Scheme for Ideal Magnetohydrodynamics", Paper 99-1677, AIAA, June 1999.

${ }^{38}$ Groth, C. P. T., De Zeeuw, D. L., Gombosi, T. I., and Powell, K. G., "Global Three-Dimensional MHD Simulation of a Space Weather Event: CME Formation, Interplanetary Propagation, and and Interaction with the Magnetosphere", J. Geophys. Res., Vol. 105, No. A11, pp. 25,053-25,078, 2000.

${ }^{39}$ Peroomian, O., Chakravarthy, S., and Goldberg, U. C., "A "Grid-Transparent" Methodology for CFD", Paper 97-0724, AIAA, June 1997.

${ }^{40}$ Peroomian, O., Chakravarthy, S., and Goldberg, U. C., "Convergence Acceleration for Unified-Grid Formulation Using Preconditioned Implicit Relaxation", Paper 98-0116, AIAA, January 1998.

${ }^{41}$ Gropp, W., Lusk, E., and Skjellum, A., Using MPI, MIT Press, Cambridge, Massachussets, 1999.

${ }^{42}$ Karypis, G., and Kumar, V., "Multilevel k-way Partitioning Scheme for Irregular Graphs", J. Parallel Dist. Comput., Vol. 48, No. 1, pp. 96-129, 1998.

${ }^{43}$ Ashkenas, H., and Sherman, F. S., "The Structure and Utilization of Supersonic Free Jets in Low Density Wind Tunnels", In Rarefied Gas Dynamics, pages 84105. Academic Press, New York, 1966.

${ }^{44}$ Crist, S., Sherman, P. M., and Glass, D. R., "Study of the Highly Underexpanded Sonic Jet", AIAA J., Vol. 4, No. 1, pp. 68-71, 1966.

${ }^{45}$ Abbet, M., "Mach Disk in Underexpanded Exhaust Plumes", AIAA J., Vol. 9, No. 3, pp. 512-514, 1971.

${ }^{46}$ Jugroot, M., Groth, C. P. T., Thomson, B., Baranov, V., and Collings, B. A., "Numerical Investigation of Neutral Flows in the Interface Region of MassSpectrometers", submitted to J. Phys. D: Appl. Phys., April 2003. 